\title{
La construcción de una ciudad con material reutilizado como escenario de stop motion. Una propuesta STEAM para educación primaria
}

STEM, STEAM, STREAM:

Posibilidades, reflexiones y experiencias

Recepción: 30/11/2020 | Revisión: 9/04/2020 | Aceptación: 05/08/2020 | Publicación: 01/10/2021

\author{
Rebeca BALSELLS-GILA \\ Universidad de Valladolid \\ rebecabalsellsgila@gmail.com \\ https://orcid.org/oooo-0oo2-3170-1147
}

\author{
María LóPEZ-LUENGO \\ Universidad de Valladolid \\ mariaantonia.lopez@uva.es \\ https://orcid.org/oooo-0oo1-7334-5278
}

\begin{abstract}
Resumen: Se presenta una contribución teórico-práctica de educación STEAM integrada. Por un lado, el marco teórico sintetiza los beneficios educativos y los retos que supone la implementación de la educación STEAM en las aulas. De acuerdo con este, se presenta una propuesta educativa diseñada para $6 .^{\circ}$ curso de educación primaria que integra contenidos de diversas disciplinas, elementos transversales y competencias curriculares. La secuencia didáctica fue valorada por 13 profesionales de la educación quienes establecieron su viabilidad y relevancia ante la imposibilidad de su implementación debido a la COVID-19. Estos destacan la selección del reto artístico para la motivación, la buena secuenciación de actividades y la gran diversidad de recursos generados para la implementación y la evaluación del proyecto.
\end{abstract}

Palabras clave: STEAM; aprendizaje basado en proyectos; stop motion; educación primaria; aprendizaje cooperativo.

\section{THE CONSTRUCTION OF A CITY WITH REUSED MATERIAL AS A STOP MOTION STAGE. A STEAM PROPOSAL FOR PRIMARY EDUCATION}

Abstract: This paper presents a theoretical and practical contribution to integrated STEAM education. On the one hand, the theoretical framework synthesises the educational benefits and challenges of implementing STEAM education in the classroom. Following this theoretical framework, an educational proposal designed for grade 6 is presented which blends content from a variety of disciplines, cross-cutting elements and curricular competencies. The didactic sequence was assessed by 13 education professionals who established its viability and relevance in view of the impossibility of its implementation due to COVID-19. The selection of the artistic challenge, the good sequencing of activities and the great diversity of resources generated for the implementation and the assessment of the learning process are highlighted by the professionals.

Keywords: STEAM; project-based learning; stop motion; primary education; cooperative learning. 


\section{LA CONSTRUCCIÓ D'UNA CIUTAT AMB MATERIAL REUTILIZAT COM ESCENARI DE STOP MOTION. UNA PROPOSTA STEAM PER A EDUCACIÓ PRIMÀRIA}

Resum: Presentem una contribució teòrico-pràctica d'educació STEAM integrada. D'una banda, el marc teòric sintetitza els beneficis educatius i els reptes que suposa la implementació de l'educació STEAM a l'aula. Seguint aquest marc, es presenta una proposa educativa dissenyada per al bè curs d'educació primària que integra continguts de diverses disciplines, elements transversals i competències curriculars. La seqüència didáctica fou valorada per 13 professionals de l'educació que van establir-ne la seva viabilitat i rellevància davant de la impossibilitat de la seva implementació degut a la COVID-19. En destaquen la selección del repte artístic per a la motivació, la bona seqüènciació d'activitats i la gran diversitat de recursos generats per a la implementació i l'avaluació del projecte. Paraules clau: STEAM; aprenentatge basat en projectes; stop motion; educació primària; aprenentatge cooperatiu.

\section{Introducción}

Las continuas y aceleradas transformaciones a las que está sometida la sociedad actual reclaman cambios educativos favorecedores de un aprendizaje adecuado y ajustado. La nueva ciudadanía requiere de competencias, capacidades de acceso, selección, interpretación y gestión de la información para abordar problemas complejos y tomar decisiones de una manera adecuada y responsable (pensamiento crítico y creativo, capacidad de colaboración, etc.).

En este sentido, las metodologías que rompen con el aprendizaje memorístico y el libro de texto como recurso único del aula están situadas en el centro de la innovación educativa. En la etapa de Educación Secundaria Obligatoria (ESO), especialmente buscando la mejora del aprendizaje científico-tecnológico, el aprendizaje basado en proyectos (ABP) y en concreto los proyectos STEM integrados han alcanzado bastante popularidad (López Secanell y Ortega Torres, 2020; Pérez-Torres et al., 2021). Sin embargo, no ha sido así en la Educación Primaria (EP), quizá por la inexistencia explícita de la tecnología y la ingeniería. La metodología STEAM tiene un enorme potencial en los distintos niveles educativos. Paulatinamente está dejando de ser desconocida, o poco valorada, en España gracias a la apuesta que han realizado algunas administraciones como la Consejería de Educación, Cultura y Deportes de Castilla la Mancha (Formación en competencias STEAM) o el Departament d'Educació de Catalunya (programa STEAMcat).

El trabajo que aquí se presenta es una contribución teórico-práctica en la línea señalada; un sustento para la realización de proyectos integrados STEAM en las aulas de EP. Se ofrece una propuesta diseñada para $6 .^{\circ}$ curso, acompañada del soporte teórico y del análisis crítico sobre su viabilidad y validez que realizó un grupo de docentes expertos. Con la propuesta educativa, se busca el logro de los objetivos de la educación primaria (concretamente $a, b, e, g, i, j$ ), mediante el trabajo explícito de contenidos curriculares de diversas disciplinas (Ciencias de la Naturaleza, Ciencias Sociales, Lengua Castellana y Literatura, Matemáticas y Educación Artística) y de las siete competencias curriculares (Comunicación lingüística; Competencia matemática y competencias básicas en ciencia y tecnología; Competencia digital; Aprender a aprender; Competencias sociales y cívicas y Sentido de iniciativa y espíritu emprendedor; Conciencia y expresiones culturales) establecidos en el Real Decreto 126/2014 (Ministerio de Educación, Cultura y Deporte, 2014). 


\section{Educación STEAM integrada}

\subsection{Definición de STEAM}

La educación STEAM se confiere como "un nuevo paradigma que plantea la Ciencia y Tecnología interpretada a través de la Ingeniería y de las Artes" (Cilleruelo y Zubiaga, 2014, p. 2). Al tratarse de un nuevo enfoque educativo, internacionalmente no existe pleno consenso en su concepción (Perignat y Katz-Buonincontro, 2019). En este trabajo se defiende como la necesidad de todos los saberes y enfoques disciplinares para el abordaje de los problemas complejos a los que nos enfrentamos en la actualidad. Por lo tanto, se entiende que la educación STEAM debe plantear al conjunto del alumnado -en su gran diversidad de aptitudes- proyectos y retos que partan de la cotidianidad de los discentes y cuyo abordaje requiera de la colaboración de todas las disciplinas. De este modo se desarrolla un proceso interdisciplinar e incluso transdisciplinar que genera un aprendizaje holístico. Se observa pues, la necesidad de considerar un formato escolar unidimensional, no fragmentado en disciplinas (Bazler y Van Sickle, 2017). Este importante requerimiento nos conduce hacia la revisión de los retos que implica la introducción de la educación STEAM en las aulas, en paralelo a los beneficios que conlleva.

\subsection{Beneficios y retos de STEAM integrado}

La investigación realizada hasta el momento muestra numerosas ventajas en la metodología STEAM, en parte debidas a que se trata de $A B P$, metodología sobradamente conocida como exitosa. En otra buena parte, los beneficios vienen dados de la integración de los aprendizajes científico-tecnológicos con los humanísticos, las artes; lo cual, entre otras ventajas, favorece la motivación y la inclusión de todo el alumnado. No obstante, algunos docentes consideran que la educación STEAM no es más que una moda pasajera (González, 2019).

La evaluación supone un reto multifacético que hay que enfrentar para llevar a cabo propuestas de educación STEAM. Al igual que sucede con propuestas de ABP y en el aprendizaje cooperativo, evaluar aprendizajes individuales cuando el trabajo es mayoritariamente grupal no es sencillo y resulta incoherente centrarse exclusivamente en el producto, si se ha puesto el acento en el proceso (Domènech-Casal et al., 2019). Por otro lado, al tratar la evaluación es fundamental discernir entre esta y la calificación (López y Pérez, 2017); el profesorado debe abordar la calificación individual y disciplinar desde la perspectiva legislativa, aunque el proceso de aprendizaje se ha realizado de modo inter o transdisciplinar.

La propuesta que se ofrecerá a continuación busca solventar tales retos metodológicos y evaluativos de la educación STEAM, sintetizados en la Tabla 1 junto a los beneficios de la misma. Como síntesis de la revisión bibliográfica realizada $y$, a partir de todo lo indicado, se ha generado una infografía que muestra algunos aspectos clave para implantar la metodología STEAM en el aula (Figura 1).

\begin{tabular}{|l|l|}
\hline \multicolumn{1}{|c|}{ VENTAJAS DE LA EDUCACIÓN STEAM } & \multicolumn{1}{|c|}{ RETOS ANTE LA EDUCACIÓN STEAM } \\
\hline $\begin{array}{l}\text { Se parte de problemas con relación al entorno que } \\
\text { rodea al alumnado posibilitando el acercamiento al } \\
\text { mundo real (Bazler y Van Sickle, 2017; Guitart y Lope, }\end{array}$ & $\begin{array}{l}\text { Dificultad y conflicto al escoger un tema específico con } \\
\text { requerimientos de la legislación educativa (González, } \\
\text { 2019) }\end{array}$ \\
$\begin{array}{l}\text { Integración de disciplinas frente a la fragmentación } \\
\text { (Segura y Caplan, 2019) }\end{array}$ & $\begin{array}{l}\text { Falta de formación del profesorado en relación con } \\
\text { esta metodología (González, 2019) }\end{array}$ \\
\hline
\end{tabular}




\begin{tabular}{|c|c|}
\hline $\begin{array}{l}\text { El enfoque STEAM integrado tiene evidencias de ser } \\
\text { una posible vía de mejora del desarrollo competencial }\end{array}$ & $\begin{array}{l}\text { Falta de correlación entre el conocimiento que tiene el } \\
\text { maestro y el tema a desarrollar en el proyecto }\end{array}$ \\
\hline $\begin{array}{l}\text { Empoderamiento de los niños y niñas en áreas } \\
\text { científico-tecnológicas (Guitart y Lope, 2019) }\end{array}$ & $\begin{array}{l}\text { La ausencia de publicaciones para el aula de Educación } \\
\text { Primaria que sirvan como ejemplo y guía a maestros } \\
\text { que se quieren iniciar en esta metodología }\end{array}$ \\
\hline $\begin{array}{l}\text { Conocimiento más profundo de ciencias y matemáticas } \\
\text { obteniendo la capacidad de integrar dicho } \\
\text { conocimiento en otras áreas (Bazler y Van Sickle, 2017) }\end{array}$ & $\begin{array}{l}\text { Preocupación por el cumplimiento del currículo y la } \\
\text { organización del centro (Domènech-Casal et al., 2019) }\end{array}$ \\
\hline $\begin{array}{l}\text { El uso de la información de manera integrada da } \\
\text { respuesta a las necesidades de nuestra sociedad, } \\
\text { trabaja destrezas innovadoras y genera autocrítica } \\
\text { (Bazler y Van Sickle,2017; González, 2019) }\end{array}$ & $\begin{array}{l}\text { Requiere de una alta coordinación y cooperación entre } \\
\text { el profesorado (Benjumeda y Romero, 2017) }\end{array}$ \\
\hline $\begin{array}{l}\text { Desarrollo de las destrezas ligadas a las artes: } \\
\text { creatividad, resolución de problemas, pensamiento } \\
\text { crítico, comunicación, autonomía, iniciativa y } \\
\text { colaboración (Cilleruelo y Zubiaga, 2014) }\end{array}$ & $\begin{array}{l}\text { Cuando el profesorado no comparte rutina diaria con } \\
\text { el alumnado y existe un constante tráfico docente } \\
\text { entre aulas, resulta casi imposible conocer los } \\
\text { intereses, motivación, fortalezas y necesidades de los } \\
\text { estudiantes (Bazler y Van Sickle, 2017) }\end{array}$ \\
\hline $\begin{array}{l}\text { Desarrollo del pensamiento convergente y divergente } \\
\text { (Bazler y Van Sickle, 2017) }\end{array}$ & $\begin{array}{l}\text { La complejidad en la evaluación de los contenidos y } \\
\text { del control grupo-clase son otros aspectos que pueden } \\
\text { dificultar al profesorado la implementación de la } \\
\text { metodología STEAM en el aula (González, 2019) }\end{array}$ \\
\hline \multicolumn{2}{|l|}{$\begin{array}{l}\text { Mejora la autoeficacia en el alumnado y del rigor } \\
\text { académico (Bazler y Van Sickle, 2017; Ortega, Verdugo } \\
\text { y Gómez, 2019). }\end{array}$} \\
\hline $\begin{array}{l}\text { Aprendizaje significativo (Benjumeda y Romero, 2017; } \\
\text { Segura y Caplan, 2019) }\end{array}$ & \\
\hline
\end{tabular}

Tabla 1. Síntesis de beneficios y retos de la de educación STEAM.

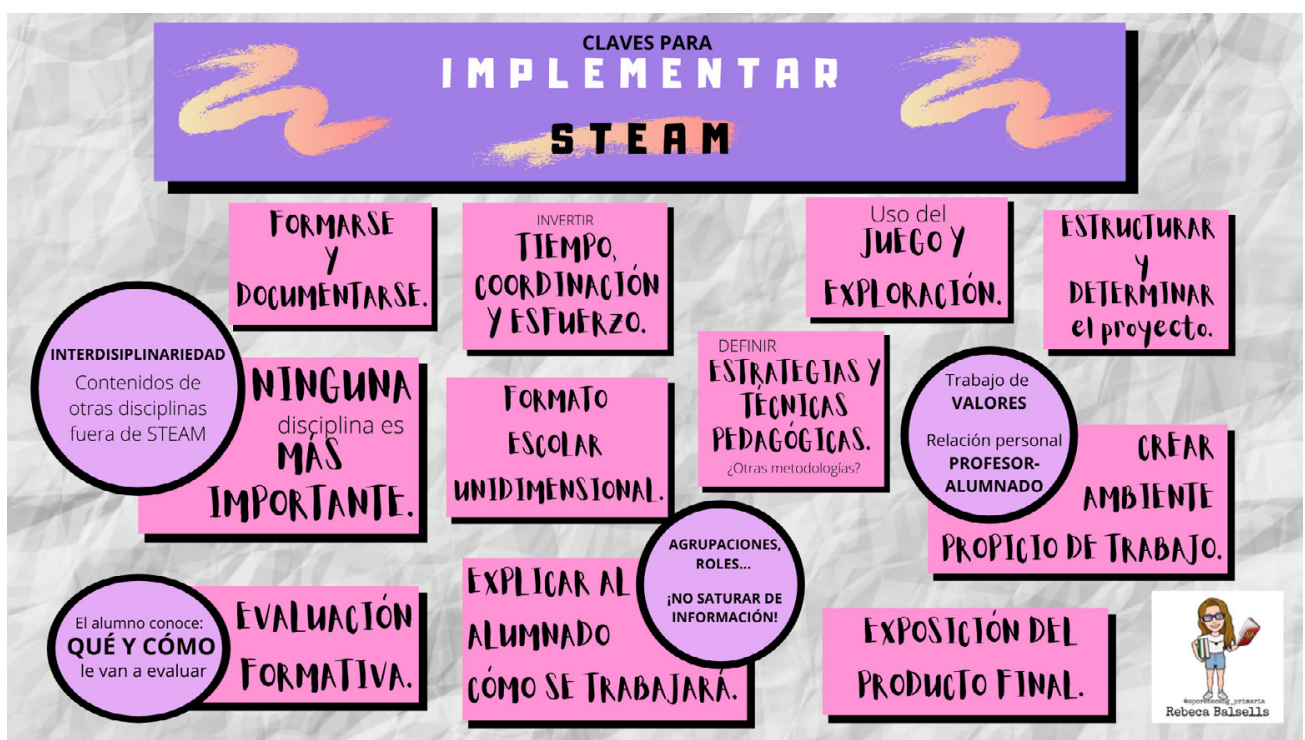

Figura 1. Claves para implementar STEAM. 


\section{Descripción de la propuesta: construcción de una ciudad con material reutilizado como escenario de Stop Motion}

Desde la responsabilidad social del docente y tomando como referencia las claves señaladas, se diseñó la propuesta que aquí se presenta, dirigida a un grupo concreto de $6 .^{\circ}$ de Educación Primaria de un colegio de Segovia. Como su implementación ha sido imposible debido a la COVID-19 se decidió someterla a un proceso de validación por expertos que se describe seguidamente.

La propuesta STEAM que se plantea constituye una parte de la primera fase de un proyecto mucho más amplio: la elaboración de un cortometraje de Stop Motion que sucede en una ciudad. Técnica cinematográfica bastante popular en la actualidad, el alumnado está familiarizado con películas (ej. Pesadilla antes de Navidad (1993)) o anuncios que utilizan esta técnica y, sin embargo, posiblemente no saben emplearla, por lo que su uso resultará altamente motivador. Esta técnica crea la ilusión de movimiento mediante la toma de imágenes sucesivas de objetos (marionetas, juguete, recortables, etc.) que se manipulan a mano (Luengo, 2012, p. 6). La creación del relato digital y del escenario tienen un gran potencial didáctico como señala Fernández y Barreira (2016). Por ello, el reto que se plantea al alumnado en este momento es la elaboración del escenario con material de desecho, como maqueta de una ciudad real. El escenario es un elemento esencial en esta técnica como en cualquier tipo de producción cinematográfica. Una vez generado de forma colectiva, permitirá la grabación de los cortometrajes de cada uno de los grupos de trabajo. Su elaboración forma parte de la primera de las tres grandes fases de un corto cinematográfico: preproducción, producción y postproducción (Lorente, 2019). El alumnado también podrá entender y valorar el trabajo que implica la realización de una película.

También realizarán una guía para elaborar un escenario de stop motion (en adelante Guía E.S.M.). Ambos productos responden a los requerimientos de un proyecto artístico: deben ser expuestos públicamente. Su elaboración requiere y pone en juego saberes científicos, de ingenieria, matemáticos, tecnológicos, además de otros incluidos en el currículo de educación primaria, pero que no constituyen parte del acrónimo STEAM como son los relacionados con el uso de la lengua.

\subsection{Secuenciación y elementos curriculares de la propuesta}

Para un correcto desarrollo del proyecto se ha llevado a cabo una organización en la que podemos diferenciar cinco bloques o fases de actividades y 8 sesiones (Tabla 2).

\begin{tabular}{|c|c|c|}
\hline \multicolumn{3}{|c|}{ ORGANIZACIÓN DEL PROYECTO } \\
\hline FASE INICIAL: ¿Escenario? ¿Qué es? & Sesión 1 & 1 hora aproximadamente. \\
\hline \multirow{3}{*}{ FASE I: Nos volvemos expertos } & Sesión 2 & 1 hora aproximadamente. \\
\hline & $\begin{array}{cl}\text { Sesión } & 3 \text { (puzzle de Aronson) } \\
\text { - } & \text { Sesión 3-Medida } \\
\text { - } & \text { Sesión 3-Materiales } \\
\text { - } & \text { Sesión 3-Construcción } \\
\text { - } & \text { Sesión 3-Diseño } \\
\text { - } & \text { Sesión 3-Guía E.S.M. }\end{array}$ & 1-2 horas aproximadamente. \\
\hline & Sesión 4 & Máximo 2 horas \\
\hline \multirow{2}{*}{$\begin{array}{c}\text { FASE II: } \\
\text { Planificamos y diseñamos nuestra } \\
\text { ciudad }\end{array}$} & Sesión 5 & No más de 2 horas y media. \\
\hline & Sesión 6 & No más de 3 horas \\
\hline FASE III: Manos a la obra & Sesión 7 & $\begin{array}{l}\text { Esta sesión podrá durar varios días } \\
\text { debido a la parte de construcción. }\end{array}$ \\
\hline FASE FINAL: Echamos la vista atrás & Sesión 8 & No más de 2 horas y media. \\
\hline
\end{tabular}

Tabla 2. Organización del proyecto. 


\begin{tabular}{|c|c|c|}
\hline \multicolumn{3}{|r|}{ ELEMENTOS CURRICULARES DE LA PROPUESTA STEAM } \\
\hline \multicolumn{2}{|c|}{ Metodología } & Educación STEAM integrado \\
\hline \multirow[t]{2}{*}{ Objetivos } & Generales & $\begin{array}{l}\text { - } \quad \text { Elaborar un escenario para las grabaciones de Stop Motion. } \\
\text { - Desarrollar un documento explicativo de cómo elaborar un escenario. para } \\
\text { Stop Motion (guía E.S.M.) }\end{array}$ \\
\hline & Específicos & - $\quad$ Adquirir contenidos curriculares de diversas asignaturas. \\
\hline \multirow[t]{6}{*}{ Contenidos } & $\begin{array}{l}\text { Science (social } \\
\text { \& natural } \\
\text { science) }\end{array}$ & $\begin{array}{l}\text { La ciudad -cómo es, vida en la ciudad, organización en barrios, edificios y sus } \\
\text { funciones-; las propiedades de los materiales; el consumo responsable: } \\
\text { reducción, reutilización y reciclaje; el medio ambiente -respeto y conservación-. }\end{array}$ \\
\hline & Technology & $\begin{array}{l}\text { El boceto -perspectivas-; las TICs para el tratamiento de imágenes, diseño y } \\
\text { animación, y su empleo para la difusión de los trabajos elaborados; manejo del } \\
\text { software de LDD como recurso para el diseño gráfico. }\end{array}$ \\
\hline & Engeneering & $\begin{array}{l}\text { La construcción de escenarios - pasos-; construcción de los edificios de la ciudad } \\
\text { mediante la unión de piezas -planificación y montaje-; protocolo de medidas de } \\
\text { seguridad; planificación y realización de proyectos y presentación de informes. }\end{array}$ \\
\hline & Arts & $\begin{array}{l}\text { Diseño gráfico; el uso de la línea y el punto; elaboración de producciones } \\
\text { tridimensionales, utilizando técnicas mixtas de elaboración, aplicadas a un fin } \\
\text { determinado. }\end{array}$ \\
\hline & Maths & $\begin{array}{l}\text { La escala y el plano; proporcionalidad en la escala de planos; representación del } \\
\text { espacio; unidades del sistema métrico decimal: longitud y superficie. }\end{array}$ \\
\hline & $\begin{array}{l}\text { Contenidos } \\
\text { comunes y de } \\
\text { otras } \\
\text { disciplinas }\end{array}$ & $\begin{array}{l}\text { El escenario como parte del cortometraje de Stop Motion; situaciones } \\
\text { comunicativas: conversaciones en grupo; estrategias y normas que rigen la } \\
\text { interacción oral; técnicas de trabajo intelectual; estructuración de la información; } \\
\text { desarrollo de estrategias para organizar, memorizar y recuperar la información, } \\
\text { recogiendo las ideas principales; la guia como texto informativo -partes y } \\
\text { funciones; el borrador como parte de la planificación del trabajo. }\end{array}$ \\
\hline
\end{tabular}

Tabla 3. Elementos curriculares del proyecto.

\subsection{Fase Inicial: ¿Escenario? ¿Qué es?}

La presentación del proyecto al alumnado es una de las partes fundamentales para el logro de los objetivos que nos proponemos como maestros. Además, el trabajo del contenido principal asentará las bases de una serie de conocimientos necesarios para una posterior y correcta aplicación de los aprendizajes adquiridos a lo largo de las restantes sesiones.

Por ello, en la primera sesión nos centramos en el trabajo del escenario como concepto. También, se asentarán las estrategias y normas que hagan posible una adecuada interacción social a lo largo de todas las sesiones. Todo esto se llevará a cabo mediante el trabajo de rutinas de pensamiento (Ritchhart et al., 2014) y la lectura reflexiva de distintos materiales diseñados y elaborados para tal fin. Además, esta sesión servirá como evaluación inicial o diagnóstico de los conocimientos previos y centros de interés del alumnado. 


\subsection{Fase I: Nos volvemos expertos}

Para que los escolares se sumerjan en la construcción del escenario tienen que adquirir y poseer unos conocimientos mínimos que les permita desarrollar al máximo sus capacidades en esta temática. Se convierten, así, en el equipo de decorado y montaje de su propia película.

En la segunda sesión se trabaja la ciudad, se desarrolla la estructuración, organización, memorización y recuperación de contenidos previos sobre este contenido. Se parte de su propia ciudad (Segovia) ampliando así su conocimiento del entorno y utilizando el plano de esta. Se utiliza la técnica de aprendizaje cooperativo "En busca de la idea global", con el apoyo de los organizadores gráficos "Los pétalos de la flor" y "La maceta", creados ad hoc debido a la inexistencia de técnicas que cubrieran los objetivos deseados. La sesión finaliza con la evaluación mediante un cuestionario individual.

Se llega así, a la tercera sesión del proyecto que se desarrolla por grupos de expertos, conformados por el docente para trabajar mediante la técnica del puzzle de Aronson (Martínez y Gómez, 2010). Distinguimos, pues, las diferentes temáticas que dan nombre a los equipos de trabajo: Medidas, Diseño, Materiales, Construcción y Guía E.S.M. Esta sesión es diferente para cada grupo y se desarrollará tanto en clase como en casa. Al inicio de la sesión, todos los grupos repartirán las tarjetas de roles y al finalizar completarán los dos primeros apartados de la "hoja de registro", la cual recoge toda la información tratada en la sesión en relación con su temática.

- El grupo de Medida: Realiza actividades sobre el plano y la escala, la proporcionalidad de estos con la realidad, la representación del espacio y las unidades del sistema métrico decimal: longitud y superficie. Para esto, se trabaja en clase la rutina de pensamiento "VeoPienso-Me Pregunto" partiendo del plano de su municipio. Las preguntas que surgen en relación a la medida y la construcción del escenario deberán ser trabajadas y respondidas individualmente en casa. En la siguiente sesión, de nuevo en los grupos de expertos, se ponen en común las respuestas y se elabora un documento de información para utilizar en la Sesión 4. La evaluación del documento se lleva a cabo mediante una rúbrica y el trabajo individual a través de un cuestionario.

- El grupo de Materiales: En clase se realiza una lluvia de ideas de manera individual sobre el material y objetos que se puede utilizar para construir el escenario, se pone en común en el grupo de expertos y se genera una nube de palabras. En casa e individualmente se repasa las propiedades de los materiales y se realiza la ficha "Identificación de objetos". De vuelta a clase, se trabaja la regla de las tres erres y el consumo responsable orientando el trabajo hacia la sostenibilidad. Para finalizar, se realiza individualmente un mapa conceptual organizando los conceptos proporcionados, un cuestionario reflexivo y un poster creativo sobre las tres erres y el consumo responsable. Para la evaluación se analizan estos últimos materiales, así como la ficha "Identificación de objetos".

- El grupo de Diseño: Trabaja en clase con la rutina de pensamiento "Veo-Me preguntoInvestigo" a partir de una imagen sobre el plano, el boceto y el diseño gráfico. En casa, individualmente, trabajarán una serie de documentos sobre el tema del boceto y el software 
Lego Digital Designer (LDD) pues será necesario para la construcción del escenario. De regreso al aula, se realizan la puesta en común de los trabajos individuales; la investigación con relación a la rutina de pensamiento; la elaboración de un póster DIN-A3 para explicar el software y la reflexión sobre la utilidad de seguir los pasos plano-boceto-diseño gráfico en la creación del escenario. Para la evaluación se utilizan las respuestas de la rutina de pensamiento, el póster grupal y las hojas sobre el boceto y el software de diseño.

- El grupo de Construcción: Trabaja en clase sobre la construcción y montaje de escenarios con las dos primeras columnas de la rutina de pensamiento "Pienso-Me interesa-Investigo" y en casa, individualmente, la tercera columna (investigar). En la siguiente sesión de aula, se ponen en común en el grupo de expertos los trabajos individuales, se reflexiona sobre un póster que muestra los pasos a seguir para hacer una maqueta (pautas que seguirán en la construcción real) y se elabora un protocolo de seguridad para el uso del material. La evaluación se realiza mediante un cuestionario que rellenan al final de la sesión, los organizadores gráficos de la rutina de pensamiento con sus investigaciones y el protocolo de seguridad creado.

- El grupo de Guía E.S.M.: En clase, y de manera grupal, realizará una lluvia de ideas sobre la estructura que puede tener una guía. A continuación, analizan varias guías proporcionadas por la docente y reflexionan sobre ellas. Individualmente, en casa el este alumnado realizará un mapa conceptual de las partes de la guía, ejemplos y la información que debe mostrar. En la siguiente sesión, el grupo elabora un borrador de la estructura de la guía y la portada definitiva sobre bocetos individuales. Para la evaluación se analizan los mapas conceptuales, el boceto de la portada, la lluvia de ideas, el borrador de estructura y la portada definitiva de la guía.

La cuarta sesión se desarrolla en los grupos de origen. En ella se irá explicando la información trabajada en cada grupo de experto y se rellenará la hoja de coevaluación cada vez que un compañero termine de exponer su tema. También se trabajará el tercer apartado de la "Hoja de registro". Tras ello, y de manera individual, se elaborará un mapa conceptual o un Visual Thinking sobre la información recibida del resto de compañeros. El alumnado regresa al grupo de expertos para poner en común la "Hoja de registro" y tomar las decisiones definitivas sobre cómo se trabajará durante la elaboración del escenario. En cada grupo de origen se genera una Iluvia de ideas sobre los edificios que tendrá la ciudad-escenario, la toma de decisiones definitiva se tendrá lugar en el gran grupo. La evaluación se realiza mediante la hoja de coevaluación y los mapas conceptuales.

\subsection{Fase II: Planificamos y diseñamos nuestra ciudad}

La planificación y diseño de la ciudad es una parte muy importante y creativa en la que los escolares deciden por consenso aquellos elementos que caracterizan su creación. Al igual que en otras fases, esta se desarrolla en varias sesiones en las que el proyecto avanza y el alumnado adquiere un aprendizaje experiencial y significativo. 
La quinta sesión de esta propuesta didáctica se inicia resolviendo un problema de optimización en asamblea: la ciudad tiene que ser modular, es decir, tienen que tener partes separables para permitir la accesibilidad a la hora de grabar; el boceto del plano debe elaborarse sobre seis tableros de pupitre cuyas medidas son $60 \mathrm{~cm}$ x $40 \mathrm{~cm}$. También, se acuerda en la asamblea qué partes del escenario son responsabilidad de cada equipo. En los grupos de origen se elabora a continuación el plano de la parte de ciudad correspondiente teniendo en cuenta la escala plano-maqueta, el perímetro de dicha parte y la superficie de los edificios a colocar. Una vez realizado, se unen los diferentes fragmentos del plano y se hace una puesta en común en gran grupo. Cada equipo trabaja la rutina de pensamiento "Titulares" sobre la sesión y se comentan en el grupo aula para generar un listado de ideas. Estos materiales se incluyen en la Guía E.S.M. Para la evaluación se hace uso del boceto completo de la ciudad, del plano de cada grupo y del plano definitivo del escenario.

La sexta sesión comienza con una asamblea para acordar los aspectos que deben incluirse en la Guía E.S.M. A continuación, la sesión se centra en el diseño de los edificios que conformarán el escenario. La mitad de cada grupo de origen se encarga de buscar información e imágenes inspiradoras para el diseño y las otras mitades de los equipos, de manera conjunta, se encargan de redactar la introducción de la Guía E.S.M. Después, con ayuda de técnicas y estrategias de creatividad, los grupos de origen elaboran bocetos de los edificios respetando cierta proporcionalidad, sin entrar en detalles, haciendo uso del punto y la línea y anotando las medidas de longitud. Una vez terminada esta actividad y de nuevo en asamblea, se repasa el funcionamiento del software de LDD con ayuda del póster DIN-A3, creado en la sesión 3, para realizar el diseño gráfico de los bocetos. En este momento sí que se entra en detalles como el color y los props ("objetos, utensilios o muebles que aparecen en el escenario" (Lorente, 2019, p. 20)). Paralelamente, se redacta el trabajo realizado para incluirlo en la Guía E.S.M. Se finaliza con la puesta en común de los diseños y el trabajo con los instrumentos de evaluación.

\subsection{Fase III: Manos a la obra}

La tercera fase del proyecto es la más manipulativa y puede alargarse varios días.

En asamblea, se comienza recordando el procolo de seguridad elaborado en la sesión 3 y se reflexiona sobre la importancia de hacer uso de material reutilizado. Los grupos de expertos Materiales y Construcción se reúnen para coordinarse y organizarse adecuadamente mientras que los demás avanzan con la elaboración de la guía. Tras ello, los escolares regresan a los grupos de origen $y$, teniendo en cuenta los bocetos y diseños gráficos de los edificios, así como las medidas correspondientes de su parte de escenario, construyen dichos edificios en una plataforma de cartón reutilizado, lo que favorece su movimiento para la grabación.

Terminada la construcción, el grupo experto Guía E.S.M. se reune para decidir la información que debe incluirse en el documento mientras que el resto analiza el resultado y propone posibles mejoras. Finalmente, todos regresan a los grupos de origen y efectúan las mejoras pertinentes. La evaluación de esta sesión se realiza mediante una escala Likert. 


\subsection{Fase final: Echamos la vista atrás}

Por fin ha llegado el momento de combinar los módulos de la ciudad completar la guía E.S.M. y disfrutar del resultado final mediante una única sesión en dos partes diferenciadas.

La primera sucede en asamblea y se centra en la composición final de la ciudad-escenario y la toma de acuerdos si fuera necesario algún cambio o mejora.

La segunda se dedica a la finalización de la Guía E.S.M. Para ello, los escolares trabajan individualmente la tercera columna del organizador de la rutina de pensamiento $\mathrm{KWL}$, realizan una puesta en común en sus equipos de trabajo y terminan de rellenar la tercera columna del organizador grupal de la misma rutina de pensamiento. Todos estos materiales se añaden al documento escrito. Después, cada escolar realiza un Visual Thinking sobre todo el proceso experimentado a lo largo de las sesiones que será evaluado por otro discente mediante una ficha de coevaluación. Finalmente, el grupo aula genera unas conclusiones sobre el proyecto que se incluirán en la guía y realiza una revisión global de la misma. De este modo el alumnado concluye su guía explicativa sobre cómo elaborar un escenario de stop motion.

\subsection{La evaluación del proyecto educativo}

La evaluación continua y formativa se considera una parte esencial del proyecto cuya inclusión se realiza de manera natural en cada sesión como ha quedado expuesto en el apartado anterior. Deseamos destacar que se busca la mejora del aprendizaje, de la labor docente y de los procesos educativos que se desarrollan en el aula día tras día (López y Pérez, 2017). Resaltamos especialmente la puesta en marcha de procesos metacognitivos que permiten el desarrollo de la competencia aprender a aprender, así como la empatía y el pensamiento crítico.

En relación con el papel docente se recomienda la realización de un diario en el que se recojan todos los aspectos relevantes a través de los cuales se posibilite un proceso de intro y retrospección sobre las actuaciones docentes conducentes a la mejora profesional.

\section{Proceso de evaluación de la propuesta por expertos}

Se llevó a cabo un proceso de investigación evaluativa sometiendo el diseño de la propuesta a un juicio experto. Al generar el grupo de expertos se buscaba una visión lo más amplia y crítica posible.

\subsection{Participantes}

Se solicitó la participación voluntaria de 20 profesores con una experiencia profesional mayor de 5 años y con gran diversidad debido a su género, procedencia geográfica y contexto socioeducativo. Finalmente, el panel quedó constituido por 13 personas tanto, profesores universitarios especialistas en formación de profesorado (6) (Pedagogía, Didáctica de las CC Experimentales, Did. de la Matemática y Did. de la Expresión Plástica), como docentes en ejercicio, mayoritariamente en la etapa de educación primaria (7). Solo 2 tenían experiencia en el diseño de proyectos STEAM. Para la valoración recibieron una carta explicativa del objetivo del trabajo que iban a realizar, un sucinto soporte teórico sobre Educación STEAM, la propuesta didáctica con los materiales y un cuestionario elaborado ad hoc. 


\subsection{Metodología}

El cuestionario anónimo estaba constituido por 10 preguntas abiertas. Las 3 primeras para situar a los evaluadores (género, nivel educativo y conocimiento de metodologías innovadoras) y las 7 siguientes para permitir la emisión de una valoración sobre el diseño y el contenido de la propuesta. Estas son las siguientes: ¿El conjunto de las actividades favorece el aprendizaje de los contenidos señalados?; ¿El conjunto de las actividades y su secuenciación favorecen el desarrollo de las competencias señaladas?; Sugerencias de mejora para la integración de conceptos o competencias; ¿El conjunto de herramientas propuestas facilita el trabajo docente y favorece el aprendizaje del alumnado de la edad a la que va dirigido?; ¿La evaluación del proceso se ha planteado de forma adecuada y con los instrumentos pertinentes?; ¿La información se presenta en la documentación de forma clara y bien organizada? Realice una valoración para cada uno de los criterios: tiempo, materiales y agrupamiento; ¿Cree que puede implementarse en cualquier aula de $6^{\circ}$ curso? ¿Por qué? ¿Cuál es mayor valor de la propuesta según su criterio?

Los participantes se codificaron mediante un número establecido sin más criterio que el orden en el que iban llegando sus aportaciones y dos letras: $\mathrm{M}$ o H (mujer/hombre) y P o M (profesor universitario/maestro). Sus aportaciones se categorizaron en las siete categorías que se muestra a continuación.

\subsection{Resultados y discusión}

Se exponen a continuación los resultados de la evaluación según las categorías establecidas.

\section{Adquisición de contenidos y competencias}

De manera general, los expertos evaluadores han indicado que las actividades favorecen el trabajo tanto de contenidos como de competencias.

Los profesores de universidad han valorado el desarrollo de aprendizajes de gran interés que no están indicados en el documento. No obstante, $5 \mathrm{HP}$ indica que no se muestra claramente la integración de los aspectos matemáticos en el propio proyecto.

Los expertos destacan las siguientes competencias: trabajo cooperativo, consciencia del propio aprendizaje a través de la organización y planificación del trabajo y su evaluación, habilidades socio-comunicativas, fomento de la reflexión, desarrollo del razonamiento y del espíritu crítico. Estos resultados son nuevas evidencias sobre el interés de la educación STEAM para el desarrollo competencial del alumnado de EP que se suman a las ya encontradas (Bazler y Van Sickle, 2017; Cilleruelo y Zubiaga, 2014; González, 2019; Ortiz, 2020).

\section{Propuestas de mejora sobre la adquisición de contenidos y competencias}

Para un mayor aprendizaje, algunos expertos sugieren trabajar el desarrollo plano de los cuerpos geométricos en la sesión de construcción de los edificios; generar más espacios para la explicación o resolución de dudas; profundizar en el trabajo con las escalas o el diseño del boceto desde distintas perspectivas pues son contenidos complejos y abstractos; alargar la temporalización. Inevitablemente, este último aspecto dependerá del grupo clase y "será la propia experiencia en el desarrollo del mismo, el que dicte qué aspectos son susceptibles de cambio y que otros necesitan reforzarse" (10HM). 


\section{Materiales generados y herramientas propuestas}

Los expertos señalan que el diseño y formato de los materiales junto con una información clara, sencilla y motivadora, fomentan el interès del alumnado. Además, según 8HM son "una gran ayuda para poder dedicarle más tiempo a la práctica de los ejercicios y registrar todo muy bien en los instrumentos". Respecto a la labor docente, se indica que las herramientas son muy valiosas para llevar a la práctica este proyecto motivante suponiendo un reto profesional interesante.

La diversidad de los materiales de soporte favorece "el aprendizaje del alumnado desde la vertiente emocional, desarrollando el pensamiento sincrético y analógico" (12HM). Además, los expertos inciden en el formato visual atractivo de los materiales y cómo el uso de las TIC y el software de Lego Digital Designer (LDD) facilita el trabajo, aunque un evaluador no ha llegado a comprender su uso. Estas cuestiones señaladas coinciden con ideas recogidas en la bibliografía referentes a la motivación, el desarrollo de destrezas, aprendizaje significativo y el empoderamiento del alumnado respecto a la ciencia y la tecnología, así como su autoeficacia (Bazler y Van Sickle, 2017; Benjumeda y Romero, 2017; Cilleruelo y Zubiaga, 2014; González, 2019; Guitart y Lope, 2019; Ortega et al., 2019; Segura y Caplan, 2019).

\section{Evaluación del proceso}

Los expertos destacan positivamente tanto el planteamiento de evaluación continua, formativa y compartida, como la diversidad de instrumentos propuestos (rúbricas, cuestionarios, tela de araña...). Estos aspectos favorecen la superación de la complejidad en la evaluación a la que se refieren González (2019) y Domènech-Casal et al. (2019). Algunos aspectos que se han destacado son: la autoevaluación del alumnado (6MM), la propuesta de co-evaluación (9MM) y que se tenga en en cuenta tanto la evaluación del profesor como la evaluación grupal e individual del alumnado (7MM).

Sin embargo, algunos evaluadores se posicionan contrariamente a la diversidad de materiales pues la falta de uniformidad en las rúbricas podría dificultar el trabajo del alumnado siendo necesaria una mayor explicación del docente. 4MP afirma que se evalúan aspectos no señalados en el apartado de objetivos y $6 \mathrm{HP}$ detecta problemes en el paso a la calificación: "en algun caso las calificaciones numéricas que asignáis en las rúbricas parecen un poco artificiales y completas -por ejemplo, 0,025 puntos".

\section{Información presentada en el documento: tiempo, materiales y agrupamiento} El diseño y la organización, así como la progresión y secuenciación de todo el proyecto global es adecuado ya que se considera que "es un gran trabajo" (4MP) y se ofrece la posibilidad de que toda la "actividad formativa se desarrolle dentro de un contexto que fomente el desarrollo de las competencias" (13HP). Por lo general, se ha realizado una correcta estimación temporal, aunque habría que centrarse en algunas sesiones concretas que pueden quedarse cortas o en dar margen para las situaciones imprevistas.

Se valora positivamente cómo se establecen los agrupamientos y los espacios de Trabajo; así mismo se resalta de este proyecto un aspecto recogido en la bibliografía (Benjumeda y Romero, 2017; Segura y Caplan, 2019) como valor de la educación STEAM: "tanto los agrupamientos como las actividades individuales permiten el fomento del trabajo cooperativo, y del proceso de aprendizaje significativo respectivamente" (8HM). No obstante, 9MM señala la importancia de tener en 
cuenta las dimensiones del aula para organizar, sobre todo, la fase III. Además, 9MM, quizá poco familiarizada con el aprendizaje cooperativo, lo ve como un riesgo: si el alumno no realiza su trabajo individual en casa dificultará el trabajo y el progreso grupal.

Los materiales propuestos se consideran pertinentes (1MP) y "bien pensados y organizados de forma que sirven para orientar y guiar al alumnado en su aprendizaje, pero le dejan un margen de acción suficientemente amplio como para que sean los protagonistas de su propio camino cognitivo" (4MP). El uso del software LDD no se llega a comprender de manera correcta.

\section{Transferibilidad de la propuesta}

Los evaluadores consideran que la propuesta puede ser implementada, sin embargo, destacan como posible problema alguno de los retos que ya se señalaron, como la falta de formación docente (González, 2019) o la necesidad de reorganización de horarios (Bazler y Van Sickle, 2017):

\footnotetext{
Podría y debería ser implementada en cualquier aula de $6^{\circ}$, si bien no en todas las aulas se dan las características para introducir este tipo de propuestas con fluidez (debido a la poca familiaridad con este tipo de metodologías procesuales). (2MP)
}

Sería deseable que una propuesta así fuera vista como viable en muchasde las aulas de $6^{\circ}$ de Primaria, aunque conllevaría una reorganización de los horarios que podría ser sencilla si es el mismo tutor quien se encarga de todas las "asignaturas" involucradas. (5HP)

También se señala la necesidad de ajustar la temporalización al grupo concreto: "Es un proyecto que puede ser implementado por otros maestros y cuya temporalización dependerá del ritmo de los alumnos" (3HP).

Hay apreciaciones contradictorias respecto a la transferibilidad de la propuesta: $8 \mathrm{HM}$ considera que "las actividades están muy bien adaptadas y pienso que permiten flexibilizar su aplicación donde haya niños/as con más dificultades físicas o intelectuales". Mientras que 4MP indica: "a lo mejor no se podría aplicar a "cualquier aula" ya que no se tiene en cuenta a las personas con diversidad funcional, aunque creo que se podría adaptar sin problema a las necesidades de cualquier persona".

\section{Lo más destacable de la propuesta}

Al señalar lo más valioso de la propuesta, los evaluadores resaltan prácticamente los mismos aspectos indicados en la bibliografía respecto a los beneficios de la educación STEAM: la constante participación del alumnado a lo largo de todo el proceso de enseñanza-aprendizaje; la conexión de las estrategias profesionales con la realidad del aula; su carácter flexible y adaptable a alumnos de diferentes ritmos y potencialidades cognitivas; el fomento del aprendizaje interdisciplinar y transversal en un solo proyecto de manera creativa y que responde a los intereses del alumnado en esta edad; la introducción de las tecnologías en el aula; el desarrollo de la creatividad; el trabajo de la motricidad fina; la esencia lúdica de construir un escenario para grabar un corto de Stop Motion, etc. 
Destacamos los comentarios de dos expertos que focalizan sobre cuestiones concretas. 2MP se centra en las habilidades y competencias:

La propuesta conecta las estrategias profesionales con la realidad del aula y fomenta la autonomía, la responsabilidad, organización y documentación, pone en valor los procesos de pensamiento, diálogo, planificación, acción y reflexión individual y conjunta, proporciona aprendizajes aplicados y globalizados que se realizan de forma progresiva y coordinada.

Mientras que $12 \mathrm{HM}$ se centra en la educación STEAM y el desarrollo sostenible:

El uso de la metodología STEAM propone un desarrollo que va a abarcar diferentes disciplinas como un todo, una visión global de la educación tan necesaria hoy en día si queremos hacer competente a nuestro alumnado. Además, el desarrollo sostenible es uno de los desafíos más importantes para el futuro y el carácter integrador de este proyecto va en consonancia con esta visión.

\section{Conclusiones}

El proceso de investigación evaluativo llevado a cabo permite afirmar el logro del objetivo propuesto: contribuir de manera teórico-práctica a la difusión e implementación de proyectos STEAM integrados en educación primaria. Los comentarios de los expertos son aportaciones relevantes para todo aquel que quiera implementar el proyecto.

Los evaluadores también señalan el logro de una propuesta transferible, integrada e integradora que consigue trabajar colaborativamente contenidos (de múltiples disciplinas), competencias, valores, destrezas y habilidades.

Según se desprende de los comentarios de los evaluadores, la propuesta educativa recoge varios elementos destacados en la bibliografía sobre el valor de la educación STEAM. Así, la construcción de una ciudad como escenario de Stop Motion se confiere como un reto para el alumnado y permite al profesorado partir del entorno e intereses delmismo. Por un lado, la temática de la ciudad permite la conexión directa con el entorno; mientras que el uso del Stop Motion resulta interesante y motivador para las y los escolares. Igualmente, favorece la mejora de las habilidades socioemocionales, la interiorización y transferencia de conocimientos, la interactividad, la empatía, la motivación intrínseca, el trabajo colaborativo/cooperativo, el emprendimiento, la coordinación con los demás, etc. 


\section{Agradecimientos}

Este trabajo emana del proyecto STEAM-CT (2019-1-BE02-KA201-060222) https://www. steam-ct.org/. Las autoras desean agradecer la generosidad de los profesores que dedicaron su tiempo a revisar, evaluar y cumplimentar los cuestionarios sobre la propuesta y los materiales diseñados.

\section{Referencias}

Bazler, J. y Van Sickle, M. (Eds.). (2017). Cases on STEAM education in practice. IGI Global.

Benjumeda F. J. y Romero, I. M. (2017). Ciudad Sostenible: un proyecto para integrar las materias científico-tecnológicas en Secundaria. Revista Eureka sobre Enseñanza y Divulgación de las Ciencias 14(3), 621-637. http://hdl.handle.net/10498/19511

Cilleruelo, L. y Zubiaga, A. (2014). Una aproximación a la Educación STEAM. Prácticas educativas en la encrucijada arte, ciencia y tecnología. Actas Jornadas de Psicodidáctica, 1-18.

https://bit.ly/2TWLmzH

Domènech-Casal J., Lope S. y Mora L. (2019). Qué proyectos STEM diseña y qué dificultades expresa el profesorado de secundaria sobre Aprendizaje Basado en Proyectos. Revista Eureka sobre Enseñanza y Divulgación de las Ciencias 16(2), 2203. https://doi.org/gqp6

Fernández-del Río, A. B. y Barreira, A. J. (2016). Storytelling: Una herramienta para la mejora de la competencia comunicativa y el pensamiento crítico con alumnado de Altas capacidades. En R. RoigVila (Ed.), Tecnología, innovación e investigación en los procesos de enseñanza-aprendizaje (pp. 422-431). Editorial Octaedro.

González, C. (2019). Una propuesta en el ámbito STEM para la enseñanza de los sistemas de ecuaciones en la ESO. Trabajo Fin de Máster. Máster Universitario en Formación del Profesorado de Educación Secundaria. Universidad de Cantabria, España. http://hdl.handle.net/10902/16782

Guitart, F. y Lope, S. (2019). Y tú, ¿te proteges del sol? Un proyecto STEM con mirada científica. Revista Eureka sobre Enseñanza y Divulgación de las Ciencias 16(3), 3202. https://doi.org/gqp8

López Pastor, V. M. y Pérez Pueyo, Á. (Coords.) (2017). Evaluación formativa y compartida en educación: experiencias de éxito en todas las etapas educativas. Universidad de León, 2017. https://buleria.unileon.es/handle/10612/5999

López Secanell, I. y Ortega Torres, E. (2020). Escape room educativa: Concepción de los futuros maestros de Educación Secundaria en especialidad de Educación Física y Tecnología sobre la experiencia de diseñar y participar en una escape room educativa. Didacticae, (8), 176-192.

Lorente, E. (2019). Palmeritas. Diseño de props, escenarios y postproducción de un cortometraje de animación stop motion. Universidad Politécnica de Valencia, España.

https://riunet.upv.es/handle/10251/126025\#

Luengo Naranjo, M. D. C. (2013). La animación Stop Motion. Técnicas y posibilidades artísticas. Cut-out: Siluetas animadas. Trabajo Fin de Máster. Máster Universitario en Postproducción Digital. Universitat Politècnica de València. http://hdl.handle.net/10251/19146

Martínez, J. y Gómez, F. (2010). La técnica puzzle de Aronson: descripción y desarrollo. En P. Arnaiz, $\mathrm{M}^{\mathrm{a}}$. D. Hurtado y F. J Soto (Coords.), 25 Años de Integración Escolar en España: Tecnología e Inclusión en el ámbito educativo, laboral y comunitario (pp. 1-6). Consejería de Educación, Formación y Empleo de Murcia. 
Ministerio de Educación, Cultura y Deporte (2014). Real Decreto 126/2014, de 28 de febrero, por el que se establece el currículo básico de la Educación Primaria BOE. 52 (sábado 1 de marzo de 2014), 19349-19420.

Ortega. E., Verdugo. J. J. y Gómez. C. B. (2019). Docente STEAM. En M. D. Soto, V. Gómez y S. Renovell. (Eds.), Rizomatrans. Educar para cambiar la mirada: hacia una cultura avanzada (130-133). Edicions Florida. https://bit.ly/3CjpRKI

Ortiz Revilla, J. (2020). El desarrollo competencial en la Educación Primaria: efectos de una propuesta STEAM integrada. Tesis doctoral. Universidad de Burgos. http://hdl.handle.net/10259/5521

Pérez-Torres, M., Couso, D. y Márquez C. (2021). ¿Cómo diseñar un buen proyecto STEM? Identificación de tensiones en la co-construcción de una rúbrica para su mejora. Revista Eureka sobre Enseñanza y Divulgación de las Ciencias. 18(1), 1301. https://doi.org/gqp7

Perignat, E. y Katz-Buonincontro, J. (2019). STEAM in practice and research: An integrative literature review. Thinking Skills and Creativity, 31, 31-43. https://doi.org/ghnscq

Ritchhart, R., Church, M. y Morrison, K. (2014). Hacer visible el pensamiento. Paidós.

Segura, W. A. y Caplan, M. (2019). Experiencias STEAM en América Latina como metodologías innovadoras de educación. https://bit.ly/3lzF1p6 